\title{
Secaba-Rank a examen: validación de su metodología sobre eficiencia en bibliotecas versus la técnica Data Envelopment Analysis (DEA) y el método Finlandia
}

\section{Testing the Secaba-Rank tool: Validating its methodology to measure the efficiency of libraries versus Data Envelopment Analysis (DEA) and the Finland method}

\author{
Pedro Lázaro-Rodríguez; Javier López-Gijón; Enrique Herrera-Viedma
}

Cómo citar este artículo:

Lázaro-Rodríguez, Pedro; López-Gijón, Javier; Herrera-Viedma, Enrique (2019). “Secaba-Rank a examen: validación de su metodología sobre eficiencia en bibliotecas versus la técnica Data Envelopment Analysis (DEA) y el método Finlandia". El profesional de la información, v. 28, n. 3, e280316.

https://doi.org/10.3145/epi.2019.may.16

Artículo recibido el 10-11-2018

Aceptación definitiva: 21-02-2019

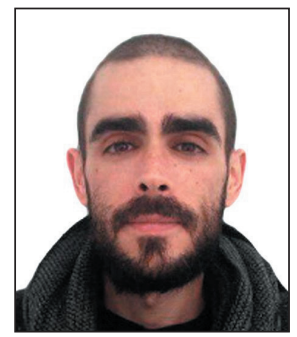

Pedro Lázaro-Rodríguez $\bowtie$ https://orcid.org/0000-0002-8756-0507

Universidad de Granada

Departamento de Información y Comunicación Campus Universitario de Cartuja

Edificio Máximo. 18071 Granada, España pedrolr@ugr.es

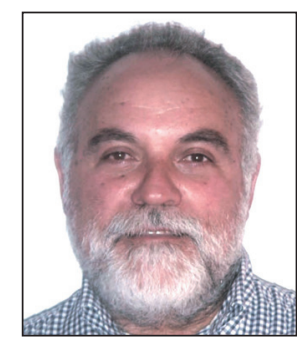
Javier López-Gijón https://orcid.org/0000-0001-6517-8991 Universidad de Granada Departamento de Información y Comunicación Campus Universitario de Cartuja Edificio Máximo. 18071 Granada, España jgijon@ugr.es

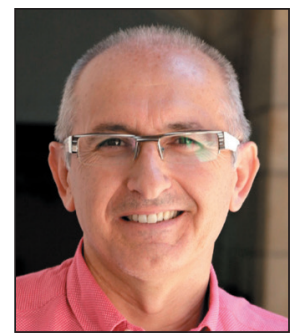

Enrique Herrera-Viedma https://orcid.org/0000-0002-7922-4984

Universidad de Granada

Departamento de Ciencias de la Computación e Inteligencia Artificial.

Periodista Daniel Saucedo Aranda, s/n. 18071 Granada, España viedma@decsai.ugr.es

\section{Resumen}

El objetivo de este artículo es probar la validez de la metodología Secaba-Rank basada en el indicador de la potencia del sistema para medir la eficiencia de bibliotecas. Para ello se ha llevado a cabo una comparación de los resultados de eficiencia según dicha metodología con los de la técnica Data Envelopment Analysis (DEA, análisis envolvente de datos) y el calificado como método Finlandia. La unidad de análisis es triple considerando a las bibliotecas públicas a nivel de comunidades autónomas y provincias, y a las bibliotecas universitarias públicas españolas. Como metodología para la comparación de los resultados de acuerdo con cada método se ha llevado a cabo un análisis basado en el cálculo de los 
coeficientes de correlación lineal para cada par de variables (métodos comparados). Los resultados hallados para todos los pares de variables son muy cercanos a 10 -1 (por la naturaleza de uno de los métodos) y con significancia estadística, por lo que puede concluirse que los diferentes métodos utilizados, incluida la metodología Secaba-Rank, miden efectivamente la eficiencia. Por último se presenta y argumenta una serie de ventajas de dicha metodología sobre y a partir de las otras dos utilizadas.

\title{
Palabras clave
}

Bibliotecas universitarias; Bibliotecas públicas; Evaluación de bibliotecas; Eficiencia; Secaba-Rank; Análisis envolvente de datos (DEA); Indicadores; Metodologías; Rankings de bibliotecas; Benchmarking.

\begin{abstract}
The purpose of this article is to validate the Secaba-Rank methodology based on the System Potency indicator to measure the efficiency of libraries. It is carried out a comparison of the efficiency results according to three methods: Secaba-Rank methodology, Data Envelopment Analysis (DEA), and the Finland method. These three methods have been used to measure the efficiency of the public libraries at the level of autonomous communities and provinces, and the Spanish public university libraries. The method used for the comparison of the results is the analysis of the linear correlation coefficients for each pair of variables. The results are very close to 1 or -1 and statistically significant in all the cases. Thus, it can be concluded that the different methods, including the Secaba-Rank methodology, measure the efficiency. Finally, some advantages for the Secaba-Rank methodology are presented and argued.
\end{abstract}

\section{Keywords}

Academic libraries; Public libraries; Library evaluation; Efficiency; Secaba-Rank; Data Envelopment Analysis (DEA); Indicators; Methodologies; Library rankings; Benchmarking.

\section{Introducción}

La eficiencia es una medida que pone en relación los recursos empleados para alcanzar un objetivo con los resultados que se quieren alcanzar (Tavares et al., 2018). En el caso de las bibliotecas se puede estudiar su eficiencia poniendo en relación su presupuesto con los resultados entendidos como los préstamos y las visitas a la biblioteca (Bustos-González, 2007). En contextos donde el presupuesto es bajo, conocer qué bibliotecas o redes de bibliotecas son las más eficientes, esto es, las que procesan mejor sus recursos en relación al presupuesto para alcanzar los máximos resultados, facilita localizar modelos de buenas prácticas para aquellas que necesitan mejorar.

En esta línea se implementó en 2016 la herramienta digital abierta Secaba-Rank que incluye una serie de rankings para conocer la eficiencia de las bibliotecas universitarias y de las redes de lectura pública españolas (Lázaro-Rodríguez et al., 2018). http://secaba.ugr.es/rank

La metodología Secaba-Rank se basa en el indicador de la potencia del sistema y en un enfoque sistémico de las bibliotecas. Las bibliotecas así entendidas son un sistema con una serie de elementos interrelacionados entre sí, a saber: las entradas (presupuesto), que son procesadas por la entidad (personal, colección, etc.), para obtener unas salidas (préstamos, visitas) y alcanzar unos beneficios (mayor capital social, humano y cultural); todo en un entorno y en un ejercicio de retroalimentación entendida como la evaluación de los sistemas para su mejora. El indicador de la potencia del sistema pone en relación las entradas y salidas para medir la eficiencia de las bibliotecas.

De acuerdo con recientes investigaciones, España tiene uno de los presupuestos más bajos en bibliotecas públicas (Lázaro-Rodríguez; Vakkari, 2018). La diferencia entre Finlandia y España para el presupuesto per cápita considerando el gasto para el personal y materiales de la colección en bibliotecas públicas por regiones fue en 2015 hasta 5,2 veces mayor en Finlandia que en España ( $38,7 €$ y $7,4 €$ respectivamente). En otro estudio reciente y en un sentido similar se pone de manifiesto la gran diferencia para los préstamos y visitas por habitantes entre España y otros países de Europa (Lázaro-Rodríguez; López-Gijón; Herrera-Viedma, 2018). España es la segunda por la cola en el ranking de préstamos por habitante con 1,30 y la séptima por la cola en el de visitas por habitante con 2,39, siempre por debajo de la media europea (4,11 y 3,04 respectivamente). España está muy lejos de Finlandia, país que marca el modelo de buenas prácticas en cuanto a uso con 17,96 préstamos por habitante, 9,73 visitas por habitante y también en lo que a beneficios en bibliotecas públicas se refiere (Sin; Vakkari, 2015; Vakkari et al., 2014; 2016).

En países como España, donde el presupuesto para bibliotecas es bajo respecto a otros países europeos, resulta importante conocer su eficiencia

Desde ahí puede entenderse la importancia de conocer la eficiencia de las bibliotecas en países como España donde el presupuesto es bajo con respecto a otros países europeos y también la importancia y el valor de Secaba-Rank como herramienta para ello. A su vez el caso de Finlandia sirve como ejemplo en alusión a la medición de la eficiencia si atendemos a su sistema estadístico para bibliotecas públicas (SYKT, 2018). Mientras que el sistema de estadísticas de bibliotecas públicas del Ministerio de Educación, Cultura y Deporte (MECD) incluye una sección en los indicadores de 
rendimiento para indicadores referidos a la eficiencia (MECD, 2018), pero que sólo se refieren al gasto y no ponen en relación dicho gasto con unos resultados (Lázaro-Rodríguez; Vakkari, 2018), el sistema finlandés incluye un indicador que pone en relación el presupuesto con los préstamos y las visitas, cuyo cálculo consiste en la división del presupuesto (suma del destinado al personal y a materiales de la colección) entre la suma de préstamos y visitas (SYKT, 2018).

En el ámbito de las bibliotecas encontramos la técnica DEA (data envelopment analysis) o análisis envolvente de datos (Charnes; Cooper; Rhodes, 1978) como método más usado para medir la eficiencia (Tavares et al., 2018). Aunque fue elaborada en el campo de la economía, a lo largo del tiempo se han llevado a cabo múltiples estudios que utilizan esta técnica para conocer la eficiencia de bibliotecas en varios países (Hammond, 2002; Chen; Morita; Zhu, 2005; Stroobants; Bouckaert, 2014; Ling-Feng; Jiung-Bin; Mu-Chen, 2014), en bibliotecas de diferentes tipos como públicas (Chen; Morita; Zhu, 2005; De-Carvalho et al., 2013) o universitarias (Chen, 1997; Reichmann; Sommersguter-Reichmann, 2006; Reichmann, 2007; Liu; Chuang, 2009; Tavares et al., 2018), y también aplicando la técnica DEA a bibliotecas españolas (Simón-De-Blas; Arias-Coello; Simón-Martín, 2007; Simon-Martín; Simon-De-Blas; Arias-Coello, 2011; 2016; Faccin-Camargo; Lobán-Acero; García-Lacalle, 2018).

En este artículo se propone someter a examen la metodología Secaba-Rank en un análisis estadístico de la relación entre los resultados de la eficiencia según dicha metodología, la técnica DEA y el método finlandés. Se plantea una comparación de resultados de acuerdo con las tres metodologías con un análisis basado en el cálculo de los coeficientes de correlación lineal para cada par. De esta forma, si las correlaciones son cercanas a 1 (o-1 por la naturaleza de uno de los métodos), se podrá concluir que la metodología Secaba-Rank es apta para medir la eficiencia de bibliotecas. Además se extraerá y argumentará una serie de ventajas de la metodología Secaba-Rank sobre la técnica DEA y el método finlandés.

Desde ahí pueden enunciarse las preguntas de investigación y los objetivos como respuesta a las mismas:

- ¿ ¿Existe correlación entre los resultados sobre eficiencia según la metodología Secaba-Rank, la técnica DEA y el método finlandés?

- ¿Es válida por tanto la metodología Secaba-Rank para medir la eficiencia en bibliotecas?

- ¿Cuáles son las ventajas de la metodología Secaba-Rank sobre las demás?

En la siguiente sección se exponen los tres métodos para medir la eficiencia, a continuación se presentan la metodología y los materiales empleados, los resultados y su interpretación, se ofrece una discusión y se finaliza con las conclusiones.

\section{Métodos para medir la eficiencia en bibliotecas}

\subsection{La metodología Secaba-Rank: el indicador de la potencia del sistema}

Este indicador pone en relación las entradas con las salidas ofreciendo información de cómo la entidad procesa las primeras (Lázaro-Rodríguez et al., 2018). Se construye a partir de indicadores de primer nivel como el presupuesto per cápita en relación con las entradas de la biblioteca y con indicadores como los préstamos de documentos per cápita relacionados con las salidas. Desde estos indicadores se calculan la potencia de entrada y la potencia de salida entendidas como la división del presupuesto per cápita de cada sistema (biblioteca o red) entre el presupuesto per cápita de la llamada base, que en Secaba-Rank corresponde a la media de todas las bibliotecas según cada nivel de análisis de los que considera, y de igual forma con la potencia de salida. La fórmula de la potencia del sistema es la siguiente:

$P($ sis $)=\frac{P(\text { sal })}{P(\text { ent })}$

Donde:

$P($ sis $)=$ Potencia del sistema

$P(s a l)=$ Potencia de salida

$P($ ent $)=$ Potencia de entrada

En la herramienta Secaba-Rank el indicador de la potencia del sistema considera en la potencia de entrada el presupuesto y en la de salida los préstamos (en el caso de Secaba-Rank Universitarias los préstamos son la suma de los domiciliarios y las descargas de los datos Counter).

http://secaba.ugr.es/rank/universitarias

Los resultados marcan tres posibles estados para las bibliotecas respecto a su eficiencia (Lázaro-Rodríguez et al., 2018), que son los de potencia, dispotencia y sin potencia (tabla 1).

Tabla 1. Estados posibles de una biblioteca según la potencia del sistema

\begin{tabular}{|l|l|l|l|}
\hline \multicolumn{1}{|c|}{ Potencia del sistema } & \multicolumn{1}{|c|}{ Descripción del sistema } & \multicolumn{1}{|c|}{ Formulación } \\
\hline $\mathrm{P}($ sis $)>1$ & Se producen más salidas que entradas se le suministran & $\mathrm{P}($ sal $)>\mathrm{P}($ ent $)$ \\
\hline $\mathrm{P}($ sis $)=1$ & Se producen las mismas salidas que entradas & $\mathrm{P}($ sal $)=\mathrm{P}($ ent $)$ & Potencia \\
\hline $\mathrm{P}($ sis $)<1$ & Se producen menos salidas que entradas se le suministran & $\mathrm{P}($ sal $)<\mathrm{P}($ ent $)$ & Dispotencia \\
\hline
\end{tabular}


Es importante incidir en el hecho de que el indicador de la potencia define tres estados posibles en los que las bibliotecas o sistemas han de encontrarse. Un sistema es:

- potente, cuando dicho indicador resulta mayor que 1 y por tanto eficiente;

- dispotente, cuando es menor que 1 y por tanto no eficiente;

- sin potencia, cuando es igual a 1, un estado en que las salidas presentan el mismo nivel que las entradas.

\subsection{El método Finlandia}

Se basa en el cálculo de un indicador que incluye el sistema de estadísticas para bibliotecas públicas de Finlandia y que se califica como de economía (SYKT, 2018). Pone en relación el presupuesto total para material de las bibliotecas (colección) y el destinado a personal con el total de préstamos y las visitas a las bibliotecas. La fórmula es la siguiente:

$E=\frac{G(p)+G(m)}{P+V}$

Donde:

$E=$ Eficiencia

$G(p)=$ gasto en personal

$G(m)=$ gastos en materiales de la colección

$P=$ total de préstamos

$V=$ total de visitas

Desde ahí los resultados sobre eficiencia basada en este indicador para las bibliotecas se interpretan como mejores cuanto más pequeños sean (SYKT, 2018).

\subsection{Técnica DEA (análisis envolvente de datos)}

La técnica DEA (Charnes; Cooper; Rhodes, 1978) consiste en una aplicación de los métodos de programación lineal empleada como medida de la eficiencia relativa de unidades organizativas con las mismas metas y objetivos (Simón-DeBlas; Arias-Coello; Simón-Martín, 2007). Tal y como exponen los autores, las unidades de análisis en el DEA son denominadas unidades de toma de decisiones DMU (decision making units). Desde ahí y como idea básica se construye una unidad organizativa modelo según la combinación de entradas y salidas de todas las analizadas. En el siguiente paso se identifica la frontera de eficiencia, de tal forma que todas las unidades organizativas que se hallen en la frontera son las que funcionan al $100 \%$ y por tanto las eficientes. Por último, la eficiencia de las restantes se calcula como relativa a las definidas como eficientes. Los valores de eficiencia de cada DMU se pueden obtener en base al modelo original conocido como CCR (por las iniciales de Charnes, Cooper y Rhodes):

$$
\operatorname{Max}_{u, v} h o=\frac{\sum_{r=1}^{s} U r \cdot Y \text { ro }}{\sum_{i=1}^{m} V i \cdot X \text { io }}
$$

S. A.:

$$
\begin{aligned}
& \frac{\sum_{r=1}^{s} U r \cdot Y \mathrm{rj}}{\sum_{i=1}^{m} V i \cdot X \mathrm{ij}} \leq \quad 1 \forall j=1 \ldots n \\
& U r, V i \geq 0 \forall r: 1 \ldots s \quad \forall i=1 \ldots m
\end{aligned}
$$

Donde:

$h_{o}$ : función objetivo. Medida de la eficiencia.

$Y_{\text {rj }}$ : output i-ésimo de la $D M U$ j-ésima siempre positivos.

$X_{\mathrm{ij}}$ : input i-ésimo de la $D M U$ j-ésima siempre positivos.

$V_{i}, U_{r}$ : soluciones del problema (ponderaciones de inputs y outputs respectivamente).

$n$ : es el número de unidades de decisión, DMU.

La técnica DEA no consiste en el cálculo de indicadores tal y como sí sucede con la metodología Secaba-Rank y el método Finlandia, sino en métodos matemáticos de programación lineal. De acuerdo con dicha técnica los valores de eficiencia quedan acotados entre 0 y 1 , siendo el 1 para la unidad o unidades organizativas que se sitúan en la frontera de eficiencia y por tanto las consideradas como eficientes (Simón-De-Blas; Arias-Coello; Simón-Martín, 2007).

La técnica DEA no consiste en el cálculo de indicadores tal y como sí sucede con la metodología Secaba-Rank y el método Finlandia 


\section{Materiales y métodos}

\subsection{Materiales}

Dado que la unidad de análisis en Secaba-Rank es triple considerando en Secaba-Rank Universitarias a las bibliotecas universitarias españolas con la posibilidad de filtrar los resultados según tipo de universidad (públicas, privadas o no presenciales), y en Secaba-Rank Públicas las bibliotecas públicas españolas con la posibilidad de filtrar los resultados a nivel de comunidades autónomas y provincias, en este trabajo se considera también una triple unidad de análisis: bibliotecas públicas por comunidades autónomas y por provincias, y bibliotecas universitarias públicas presenciales.

http://secaba.ugr.es/rank/publicas

Para la variable de entrada en el indicador de la potencia del sistema de la metodología Secaba-Rank y en la técnica DEA se considera lo contemplado en el indicador finlandés, a saber, el presupuesto destinado al personal y a los materiales de la colección. Igualmente como variables de salida se incluyen las visitas y los préstamos en los tres métodos. Para el caso del indicador de la potencia del sistema de la metodología Secaba-Rank este hecho supone dar un paso más, pues originalmente dicho indicador sólo incluía los préstamos en la variable de salida (Lázaro-Rodríguez et al., 2018). Por ello se considerará en los resultados un apartado que compare los resultados de la metodología Secaba-Rank con los de la técnica DEA considerando sólo los préstamos en la variable de salidas. A su vez cabe recordar que en el caso de las bibliotecas universitarias los préstamos incluyen las descargas de las bases de datos (Ilamados datos Counter) además de los préstamos domiciliarios. Por último los datos para la población en la metodología Secaba-Rank corresponden a los de cada comunidad autónoma y provincia según el nivel analizado en el caso de las bibliotecas públicas y a la suma de estudiantes de grado, posgrado y títulos propios, y la suma de docentes a tiempo completo y parcial en el caso de las bibliotecas universitarias.

Los datos que se utilizan corresponden al año más reciente disponible, siendo el 2016 para el caso de las bibliotecas públicas tanto a nivel de comunidades autónomas como de provincias y el 2017 para las bibliotecas universitarias públicas. Para las públicas los datos han sido tomados de la web Bibliotecas públicas españolas en cifras (MECD, 2018) y de la Red de Bibliotecas Universitarias (Rebiun) para las universitarias (Rebiun, 2018). Cabe señalar que en el caso de las bibliotecas universitarias públicas se han incluido 44 bibliotecas de las 48 totales en Rebiun por falta de datos para alguna de las variables consideradas en este trabajo. Los datos fueron descargados de ambas fuentes el día 4 de octubre de 2018.

De acuerdo con lo anterior se configuraron tres conjuntos de datos: para las bibliotecas públicas por comunidades autónomas, por provincias, y para las 44 bibliotecas universitarias públicas presenciales con los datos requeridos disponibles. https://osf.io/7p2r9

https://osf.io/qr7wj

https://osf.io/csmbg

Por último y en cuanto a materiales empleados cabe mencionar que tanto el procesamiento de los datos como el cálculo de indicadores en los casos de la metodología Secaba-Rank y el indicador finlandés se ha hecho con la hoja de cálculo Libreoffice Calc 6.0.5.2. Por su lado los cálculos para la técnica DEA se han realizado con el software de código abierto OSDEA-GUI 0.2.

http://opensourcedea.org/download-osdea-gui

\subsection{Metodología}

El estudio de la relación de los resultados de la eficiencia medida según los tres métodos expuestos en el punto anterior se plantea mediante un análisis basado en el cálculo del coeficiente de correlación lineal para comparar los resultados según cada método. En primer lugar se toman los datos para su procesamiento y el cálculo de los indicadores en el caso de la metodología Secaba-Rank y el indicador finlandés, y también se importan al software mencionado en la sección anterior para el cálculo según la DEA. Dado que se utilizan 3 conjuntos de datos los resultados también se clasifican en tres conjuntos para las bibliotecas públicas a nivel de comunidades autónomas, de provincias y las bibliotecas universitarias públicas presenciales. Por último se calculan los coeficientes de correlación lineal entre los resultados de las tres metodologías.

https://osf.io/34vq9

https://osf.io/c5yjd

https://osf.io/2gacu

A este respecto y tal y como se expuso en la sección de materiales, se planteará una primera comparación de los resultados entre la metodología Secaba-Rank y la técnica DEA considerando en la variable salidas sólo los préstamos. La segunda comparación será triple entre los resultados según las dos metodologías anteriores y el indicador de Finlandia, ahora ya incluyendo también las visitas en las salidas.

Por tanto en total se tienen hasta 5 métodos diferentes (tabla 2), y todo según los 3 niveles de análisis (bibliotecas públicas por comunidades autónomas y provincias, y bibliotecas universitarias). 
Tabla 2. Abreviaturas y explicación de los 5 métodos utilizados

\begin{tabular}{|l|l|}
\hline Método & Explicación \\
\hline Psis1 & Potencia del sistema con préstamos en salidas \\
\hline DEA1 & Técnica DEA con préstamos en salidas \\
\hline Psis2 & Potencia del sistema con préstamos y visitas en salidas \\
\hline DEA2 & Técnica DEA con préstamos y visitas en salidas \\
\hline Finlandia & Indicador de Finlandia que incluye préstamos y visitas en salidas \\
\hline
\end{tabular}

Sobre el método nombrado como Psis2 cabe decir que puede entenderse desde la siguiente fórmula:

$P($ sis $)=\frac{(P(\text { salP })+P(\text { salv })) / 2}{P(\text { ent })}$

Donde:

$P($ sis $)=$ Potencia del sistema

$P($ salP $)=$ Potencia de salida referida a los préstamos

$P(s a / V)=$ Potencia de salida referida a las visitas

$P($ ent $)=$ Potencia de entrada

En este caso los préstamos y las visitas tendrían el mismo peso, pero si se quisiera dar más peso a alguna de las variables, bastaría con multiplicarla por el porcentaje deseado y eliminar la división por 2 para ambas variables en el numerador de la fórmula.

La comparación de rankings y resultados en la disciplina de la documentación se ha llevado a cabo en diversos trabajos en estudios sobre identificación de documentos altamente citados en varias bases de datos (Martín-Martín et al., 2016; Martín-Martín et al., 2017), comparando los resultados en la web en diferentes buscadores (Bar-Ilan, 2005), analizando la correlación de rankings de universidades para la creación de un meta-ranking (Luque-Martínez; Faraoni; Doña-Toledo, 2018), o comparando los resultados de diferentes factores de impacto en revistas científicas (Elkins et al., 2010).

Para el cálculo de los coeficientes de correlación lineal se ha estudiado primero la normalidad de las variables según el test de Shapiro-Wilks, utilizando el coeficiente de correlación lineal de Pearson o el de Spearman según la existencia o no de normalidad respectivamente (Fernández; Rubio, 2013). Cuando al menos una de las variables de cada par no sigue una distribución normal, se ha utilizado el coeficiente de Spearman, y si las dos eran normales, el de Pearson. Los resultados para el p valor en el test de Shapiro-Wilks con la determinación de la normalidad de las variables se muestra en la tabla 3.

Tabla 3. Normalidad y $\mathrm{p}$ valor de las variables por nivel de análisis

\begin{tabular}{|l|c|c|c|c|c|c|}
\hline \multirow{2}{*}{ Método } & \multicolumn{2}{|c|}{ Comunidades autónomas } & \multicolumn{2}{c|}{ Provincias } & Universitarias \\
\cline { 2 - 7 } & p valor & Normalidad & p valor & Normalidad & Normalidad \\
\hline Psis1 & .511 & sí & sí & 0,000 & no & 0,066 \\
\hline DEA1 & .489 & sí & 0,002 & no & 0,063 \\
\hline Psis2 & .084 & sí & 0,009 & no & 0,359 \\
\hline DEA2 & .168 & sí & 0,327 & sí & 0,178 & 0,005 \\
\hline Finlandia & .583 & sí & no \\
\hline
\end{tabular}

Por tanto en el caso de las bibliotecas públicas por comunidades autónomas y donde todas las variables son normales, se ha usado el coeficiente de Pearson. En los casos por provincias y dado que sólo se da normalidad en el caso del indicador finlandés, las correlaciones se han calculado en base al coeficiente de Spearman. Y para las bibliotecas universitarias públicas, dado que no se da normalidad en los resultados para el indicador finlandés, las correlaciones que implican dicha variable se han calculado con el coeficiente de Spearman y las restantes con el de Pearson.

Por último cabe mencionar que los cálculos estadísticos se realizaron con el software IBM SPSS 24.0 Desktop Linux Client Multilingual.

\section{Resultados}

Los resultados en este trabajo se refieren a los del análisis de las correlaciones entre los resultados de medir la eficiencia de acuerdo con los métodos y técnicas planteados. Pese a que no se presentan los de la eficiencia de las bibliotecas según cada nivel de análisis, a modo de ejemplo se añaden en la tabla 4 los resultados de la
Las comunidades autónomas obtienen la misma posición en los rankings de eficiencia medida según la potencia del sistema y la técnica DEA 
eficiencia para las bibliotecas públicas españolas a nivel de comunidades autónomas calculados según el indicador Psis1 y la técnica DEA1.

Tal y como se observa gracias a la columna para la posición de cada comunidad autónoma en el ranking de eficiencia de acuerdo con Psis1 y DEA1, todas obtienen la misma posición según cada método. Además también se observa claramente cómo para los resultados DEA1 la Comunidad Foral de Navarra sería la comunidad autónoma eficiente y a partir de la cual se han calculado las eficiencias relativas para el resto. En cambio para el caso Psis1 las redes eficientes serían las que obtienen un resultado mayor que 1 , y las dispotentes las restantes, no dándose ningún caso de sin potencia (resultado igual a 1). El análisis de la relación de los resultados es el que se plantea con el cálculo de las correlaciones. En ese sentido los resultados para las correlaciones en base al indicador Psis1 y DEA1 pueden verse en la tabla 5.

Los resultados son de correlación total en las bibliotecas públicas por comunidades autónomas y en las bibliotecas universitarias públicas, y tan sólo de una centésima por debajo del 1 en las bibliotecas públicas por provincias. Además hay significación estadística al 0,01 en todos los casos, lo que permite asegurar que hay relación lineal entre las variables. Por tanto se puede decir que los resultados de cada biblioteca (a nivel de comunidades autónomas, provincias, y las universitarias) en los rankings resultantes para cada caso por nivel de análisis son muy similares en todos los casos según el indicador Psis1 y DEA1.

Los resultados de las correlaciones entre los métodos Psis2, Finlandia y DEA2 se muestran en la tabla 6.

Los resultados en todos los pares de variables son cercanos a $10-1$. A este respecto cabe recordar que en el método Finlandia los mejores resultados en cuanto a eficiencia son los más bajos, de ahí que las correlaciones con los otros dos métodos sean negativas pues en cambio en esos dos casos las bibliotecas más eficientes son las que obtienen el valor más alto. El resultado para la correlación más lejano de 1 (o -1 en el caso de correlaciones negativas) se da en el par de variables de las bibliotecas públicas a nivel de provincias y los resultados generados a partir de Psis2 y DEA2. Aun así el resultado es de 0,841, y por ello puede decirse que la correlación es cercana a ser muy alta. El resto de resultados se mueve entre el 0,932 y el 0,980 en el caso de las correlaciones positivas y entre el $-0,933$ y $-0,985$ en el caso de las negativas. Además hay de nuevo significación estadística al 0,01 con relación lineal entre las variables.

Como ejemplo ilustrativo del anterior análisis de las correlaciones se incluyen en la tabla 7 los resultados de eficiencia para las bibliotecas universitarias públicas según los tres métodos considerados.
Tabla 4. Eficiencia según Psis1 y DEA1 para bibliotecas públicas por comunidades autónomas

\begin{tabular}{|l|c|c|c|}
\hline Comunidad Autónoma & Psis1 & DEA1 & Posición* \\
\hline Comunidad Foral de Navarra & 1,54 & 1 & 1 \\
\hline Castilla y León & 1,41 & 0,91 & 2 \\
\hline La Rioja & 1,38 & 0,90 & 3 \\
\hline Principado de Asturias & 1,33 & 0,86 & 4 \\
\hline Catalunya & 1,30 & 0,84 & 5 \\
\hline Aragón & 1,20 & 0,78 & 6 \\
\hline Illes Balears & 1,14 & 0,74 & 7 \\
\hline Región de Murcia & 0,98 & 0,64 & 8 \\
\hline Comunidad Valenciana & 0,95 & 0,62 & 9 \\
\hline Cantabria & 0,92 & 0,59 & 10 \\
\hline Castilla-La Mancha & 0,91 & 0,59 & 11 \\
\hline Comunidad de Madrid & 0,89 & 0,58 & 12 \\
\hline Galicia & 0,85 & 0,55 & 13 \\
\hline Extremadura & 0,84 & 0,55 & 14 \\
\hline País Vasco & 0,76 & 0,50 & 15 \\
\hline Canarias & 0,62 & 0,40 & 16 \\
\hline Andalucía & 0,58 & 0,38 & 17 \\
\hline
\end{tabular}

Nota: aunque los resultados se presentan con 2 decimales, se han ordenado utilizando todos los decimales obtenidos en su cálculo.

* Coincide la misma de acuerdo con los 2 métodos Psis1 y DEA1

Tabla 5. Coeficiente de correlación lineal entre Psis1 y DEA1 por nivel de análisis

\begin{tabular}{|l|c|}
\hline Nivel de análisis & Psis1-DEA1 \\
\hline CCAA & $1,000^{* *}$ \\
\hline Provincias & $0,999^{* *}$ \\
\hline Universitarias & $1,000^{* *}$ \\
\hline
\end{tabular}

$* * p \leq 0,01$ de análisis

\begin{tabular}{|l|c|c|c|}
\hline Nivel de análisis & Psis2-Finlandia & Psis2-DEA2 & DEA2-Finlandia \\
\hline CCAA & $-0,960^{* *}$ & $0,932^{* *}$ & $-0,933^{* *}$ \\
\hline Provincias & $-0,960^{* *}$ & $0,841^{* *}$ & $-0,945^{* *}$ \\
\hline Universitarias & $-0,985^{* *}$ & $0,980^{* *}$ & $-0,959^{* *}$ \\
\hline
\end{tabular}

$* * p \leq 0,01$

Las posiciones de cada biblioteca universitaria pública son muy similares de acuerdo con los tres métodos empleados para medir la eficiencia
Tabla 6. Coeficiente de correlación lineal entre Psis2, DEA2 y Finlandia por nivel 
Tabla 7. Eficiencia según Psis2, Finlandia y DEA2 para bibliotecas universitarias públicas

\begin{tabular}{|c|c|c|c|c|}
\hline Biblioteca & Psis2 & Finlandia & DEA2 & Posiciones* \\
\hline U. de Córdoba & 1,67 & 1,47 & 1,00 & $1 / 1 / 1$ \\
\hline U. Politècnica de València & 1,53 & 1,69 & 0,99 & $2 / 3 / 2$ \\
\hline U. Jaume I & 1,52 & 1,65 & 0,93 & $3 / 2 / 3$ \\
\hline U. Autònoma de Barcelona & 1,49 & 1,77 & 1,00 & $4 / 5 / 1$ \\
\hline U. de Sevilla & 1,44 & 1,76 & 0,90 & $5 / 4 / 5$ \\
\hline U. Pablo de Olavide & 1,39 & 1,87 & 0,91 & $6 / 7 / 4$ \\
\hline U. Pompeu Fabra & 1,31 & 1,91 & 0,80 & $7 / 8 / 8$ \\
\hline U. de Málaga & 1,30 & 1,86 & 0,84 & $8 / 6 / 6$ \\
\hline U. de València & 1,24 & 2,10 & 0,80 & $9 / 10 / 7$ \\
\hline U. Autónoma de Madrid & 1,18 & 2,26 & 0,80 & $10 / 14 / 9$ \\
\hline U. de Barcelona & 1,17 & 2,23 & 0,76 & $11 / 12 / 11$ \\
\hline U. de Girona & 1,16 & 2,08 & 0,76 & $12 / 9 / 10$ \\
\hline U. Rovira i Virgili & 1,14 & 2,25 & 0,73 & $13 / 13 / 14$ \\
\hline U. de Cádiz & 1,10 & 2,39 & 0,72 & $14 / 19 / 15$ \\
\hline U. Politécnica de Cartagena & 1,09 & 2,20 & 0,74 & $15 / 11 / 13$ \\
\hline U. Carlos III de Madrid & 1,08 & 2,29 & 0,65 & $16 / 15 / 19$ \\
\hline U. de Alcalá & 1,05 & 2,34 & 0,63 & $17 / 16 / 21$ \\
\hline U. de Cantabria & 1,04 & 2,36 & 0,64 & $18 / 18 / 20$ \\
\hline U. Castilla-La Mancha & 1,03 & 2,35 & 0,66 & $19 / 17 / 18$ \\
\hline U. de Almería & 1,02 & 2,58 & 0,68 & $20 / 21 / 16$ \\
\hline U. del País Vasco & 1,02 & 2,66 & 0,74 & $21 / 23 / 12$ \\
\hline U. Politècnica de Catalunya & 0,96 & 2,64 & 0,61 & $22 / 22 / 25$ \\
\hline U. de Murcia & 0,95 & 2,56 & 0,61 & $23 / 20 / 24$ \\
\hline U. de Granada & 0,95 & 2,73 & 0,61 & $24 / 26 / 23$ \\
\hline U. de Oviedo & 0,91 & 2,98 & 0,68 & $25 / 31 / 17$ \\
\hline U. de Lleida & 0,90 & 2,75 & 0,54 & $26 / 27 / 31$ \\
\hline U. de Jaén & 0,89 & 2,87 & 0,57 & $27 / 29 / 26$ \\
\hline U. de Salamanca & 0,89 & 2,72 & 0,57 & $28 / 24 / 27$ \\
\hline U. de Zaragoza & 0,88 & 2,78 & 0,54 & $29 / 28 / 30$ \\
\hline U. Rey Juan Carlos & 0,87 & 2,73 & 0,61 & $30 / 25 / 22$ \\
\hline U. de León & 0,87 & 2,96 & 0,56 & $31 / 30 / 28$ \\
\hline U. Miguel Hernández & 0,74 & 3,52 & 0,48 & $32 / 36 / 32$ \\
\hline U. Politécnica de Madrid & 0,73 & 3,44 & 0,46 & $33 / 34 / 34$ \\
\hline U. Pública de Navarra & 0,73 & 3,39 & 0,44 & $34 / 32 / 36$ \\
\hline U. de Extremadura & 0,71 & 3,41 & 0,46 & $35 / 33 / 33$ \\
\hline U. da Coruña & 0,69 & 3,80 & 0,45 & $36 / 37 / 35$ \\
\hline U. Internacional de Andalucía & 0,65 & 3,50 & 0,55 & $37 / 35 / 29$ \\
\hline U.d'Alacant & 0,65 & 3,88 & 0,40 & $38 / 38 / 38$ \\
\hline U. de La Rioja & 0,62 & 4,13 & 0,39 & $39 / 40 / 39$ \\
\hline U. de Valladolid & 0,60 & 4,00 & 0,41 & 40 / $39 / 37$ \\
\hline U. de Las Palmas de Gran Canaria & 0,59 & 4,36 & 0,37 & $41 / 41 / 41$ \\
\hline U. de Huelva & 0,55 & 4,86 & 0,39 & $42 / 43 / 40$ \\
\hline U. de La Laguna & 0,55 & 4,68 & 0,35 & $43 / 42$ / 42 \\
\hline U. de Santiago de Compostela & 0,46 & 5,86 & 0,34 & $44 / 44 / 43$ \\
\hline
\end{tabular}

Nota: aunque los resultados se presentan con 2 decimales, se han ordenado utilizando todos los decimales obtenidos en su cálculo.

*Primer valor para la posición según Psis2, el segundo según método Finlandia, y el tercero según DEA2.

A partir de la tabla 7 y considerando que en el caso de los resultados y posiciones según la técnica DEA2 hay 2 bibliotecas consideradas como eficientes con un resultado de 1 , las posiciones de cada biblioteca son muy similares de acuerdo con cada uno de los tres métodos empleados. De ahí que los resultados presentados anteriormente en la tabla 6 referidos a las bibliotecas universitarias públicas anterior sea para el par de variables Psis2-Finlandia de -0,985, de 0,980 en el par Psis2-DEA2 y en el caso DEA2-Finlandia de -0,959. 
También se puede comprobar volviendo a la tabla 7 que según los resultados para DEA2 las bibliotecas eficientes serían las de la Universidad de Córdoba y la Universitat Autònoma de Barcelona (resultado de 1). Desde ahí se obtienen los resultados relativos de las demás. En cambio en los resultados según Psis2 las bibliotecas eficientes llegarían a un total de 21 , justo las que quedan por encima de 1 y cuyo estado sería el de potencia. Por su lado en los resultados según el método Finlandia las mejores bibliotecas serían las que obtienen los valores más bajos en dicho indicador.

\section{Discusión}

Los objetivos en este trabajo fueron planteados como las respuestas a tres preguntas de investigación. Las dos primeras se referían al análisis de la correlación entre los resultados para medir la eficiencia en bibliotecas de mediante tres métodos. En cuanto a la primera pregunta de investigación lo alcanzado en el trabajo permite concluir que existe correlación entre los resultados sobre eficiencia según la metodología Secaba-Rank, la técnica DEA y el método finlandés. Como se ha mostrado, las correlaciones siempre resultaron muy próximas a 1 y -1 y siempre con significancia estadística.

El estudio de las correlaciones se propuso en la dirección que marca el título del trabajo y que consistió en someter a examen a la metodología Secaba-Rank basada en el indicador de la potencia del sistema frente a la técnica DEA y el nombrado como método Finlandia para medir la eficiencia. Con todo y en relación a la segunda pregunta de investigación, los resultados de este trabajo permiten concluir que la metodología Secaba-Rank es válida para medir la eficiencia en bibliotecas.

En cuanto a la tercera pregunta de investigación en relación con las posibles ventajas de la metodología Secaba-Rank sobre y a partir de las demás, y con la tesis de López-Gijón (2003) donde se presentó originalmente el indicador de la potencia del sistema, puede enumerarse una serie de ventajas:

- Valor de normalidad y estados de las bibliotecas en cuanto a su eficiencia: tal y como se vio en la tabla 1, los resultados del indicador de la potencia del sistema se pueden clasificar según los estados de potencia (mayor que 1), dispotencia (menor que 1), y sin potencia (igual a 1). El resultado igual a 1 marca una normalidad y es cuando la potencia de salida es igual a la de entrada. Cuando la potencia de salida es mayor que la de entrada (mayor que 1) la biblioteca o el sistema es eficiente; y cuando la potencia de salida es menor que la de entrada (resul-

Las bibliotecas han de encontrarse necesariamente en alguno de los tres estados nombrados: o son potentes, o dispotentes, o sin potencia tados menores que 1), no es eficiente. Así se puede obtener un conjunto de bibliotecas eficientes (con potencia), no eficientes (dispotentes) y sin potencia, algo que no ocurre en el método de Finlandia.

Con todo, las bibliotecas han de encontrarse necesariamente en alguno de los tres estados nombrados: o son potentes, o dispotentes, o sin potencia.

- Eficiencia relativa a la base o a las unidades organizativas en la frontera de eficiencia: tal y como se vio en los resultados y puede comprobarse en los conjuntos de datos referenciados en la metodología, mientras que la técnica DEA define tan sólo como eficientes a aquellas unidades organizativas que se sitúan en la frontera de eficiencia y que en los casos analizados mostrados en las tablas 4 y 7 han sido como mucho de hasta 2 bibliotecas, la metodología Secaba-Rank ha ofrecido siempre un mayor número de bibliotecas eficientes. Además la eficiencia del resto de bibliotecas según la técnica DEA es una eficiencia relativa a las definidas como eficientes. En cambio en el caso del indicador de la potencia del sistema la eficiencia de las bibliotecas es calculada como relativa a la considerada como base (media de todas las bibliotecas en cada nivel de análisis), posibilitando que el número de bibliotecas eficientes en la metodología Secaba-Rank sea mayor. Pero también si se considerase en el cálculo de la potencia del sistema como base los resultados para el presupuesto per cápita, y los préstamos y visitas per cápita de una biblioteca cualquiera en el cálculo de la potencia de entrada o de salida respectivamente, se obtendrían unos resultados de eficiencia relativos a esa biblioteca considerada como base. Por ello puede decirse que la metodología Secaba-Rank es capaz de adaptarse a la técnica DEA, pero no al revés. Además

La metodología Secaba-Rank es capaz de adaptarse a la técnica DEA, pero no al revés

lo referido en esta ventaja no se da en el caso del indicador finlandés pues este no marca un resultado de normalidad y sólo marca como precepto de interpretación que los resultados más bajos son los mejores en cuanto a eficiencia.

- Capacidad de incluir nuevas variables y aplicabilidad a cualquier grupo de unidades organizativas: tal y como sucede con la técnica DEA, en el caso del indicador de la potencia del sistema se ha probado su grado de adaptabilidad con la inclusión de nuevas variables en las salidas, pues el indicador Psis1 incluía sólo los préstamos y el indicador Psis2 añadía las visitas. Este hecho implica que en el indicador de la potencia del sistema se podrían incluir las variables de entrada y salida deseadas en nuevas medidas de eficiencia. También y siguiendo el ejemplo de la técnica DEA, puede decirse que el uso de la potencia del sistema no sólo quedaría reducido a medir la eficiencia en bibliotecas si no que se podría usar para medirla en cualquier tipo de unidad organizativa.

En el indicador de la potencia del sistema se podrían incluir las variables de entrada y salida deseadas en nuevas medidas de eficiencia 
- Modelos de buenas prácticas y benchmarking: del indicador de la potencia del sistema se deriva el de la formulación del sistema. Dicha formulación ofrece información muy sintética sobre cómo procesan los sistemas de biblioteca las entradas al tratar la entidad como si fuese una caja negra (Lázaro-Rodríguez et al., 2018). Desde ahí y ordenando los resultados según la potencia de entrada, pueden conocerse los sistemas que tienen entradas similares y analizar las salidas que consiguen unos y otros. Todo ello facilita la identificación de los modelos de buenas prácticas y el benchmarking para la mejora de los sistemas de biblioteca que más lo necesitan. Mientras que en la técnica DEA los modelos de buenas prácticas son los considerados como eficientes, en la metodología Secaba-Rank pueden considerarse como modelo de buenas prácticas según la ordenación por potencia de entrada los que, teniéndola similar, obtienen una mayor potencia de salida en la comparación.

- Sencillez, precisión y economía en el desarrollo de nuevos indicadores: la norma ISO 11620:2014 sobre indicadores de rendimiento en bibliotecas establece como criterios para diseñar nuevos indicadores, la sencillez y precisión de su formulación y la economía de recursos en los procedimientos de obtención de los datos (ISO, 2014). En ese sentido y dado que la metodología Secaba-Rank se basa en indicadores de primer nivel como el presupuesto, préstamos y visitas per cápita para el cálculo de la potencia del sistema de segundo nivel, puede entenderse que se ajusta en mayor grado que la técnica DEA basada en métodos matemáticos de programación lineal a los criterios de la norma ISO mencionada.

\section{Conclusión}

Este trabajo supone la prueba de que la metodología Secaba-Rank basada en el indicador de la potencia del sistema para medir la eficiencia de bibliotecas es tan válida como lo son la técnica DEA y el método Finlandia. El estudio de las correlaciones entre los resultados de acuerdo con los tres métodos permite concluir que las correlaciones son muy altas y con significación estadísti-

\section{La metodología Secaba-Rank basada en el indicador de la potencia del sistema para medir la eficiencia de bibliotecas es tan válida como la técnica DEA y el mé- todo Finlandia}

ca. Además se ha aportado una serie de ventajas que pueden atribuirse a la metodología Secaba-Rank a partir y sobre las demás. En ese sentido la originalidad del trabajo radica en haber sometido a examen la metodología Secaba-Rank frente a métodos y técnicas tan populares en el ámbito científico como la DEA para la medición de la eficiencia.

La comparación de los métodos se planteó partiendo de los niveles que contempla Secaba-Rank y fueron los de las bibliotecas públicas españolas a nivel de comunidades autónomas y provincias, y las bibliotecas universitarias públicas. Es justo lo que puede marcarse como limitación principal por cuanto la comparación se reduce a dichos niveles y a bibliotecas españolas. No obstante, las limitaciones de una investigación marcan también los posibles puntos de partida para futuros trabajos de investigación y este estudio puede ser el ejemplo y base de futuras comparaciones de métodos para medir la eficiencia que incluyan la metodología Secaba-Rank y la técnica DEA en bibliotecas de otros países y de diverso tipo.

Por último y en cuanto a las implicaciones o perspectivas de futuro, gracias a los buenos resultados hallados en este trabajo, Secaba-Rank podrá incluir de manera justificada en futuras actualizaciones de sus rankings las visitas junto con los préstamos en la potencia de salida. A su vez y dado que la técnica DEA se ha aplicado desde su origen a unidades organizativas de diversa naturaleza y no sólo a bibliotecas, podrían plantearse investigaciones aplicando el indicador de la potencia del sistema a otras unidades organizativas diferentes de las bibliotecas para medir y conocer su eficiencia.

\section{Referencias}

Bar-Ilan, Judit (2005). "Comparing rankings of search results on the Web". Information processing \& management, v. 41, n. 6, pp. 1511-1519.

https://doi.org/10.1016/j.ipm.2005.03.008

Bustos-González, Atilio (2007). “Bibliotecas universitarias: ¿sabemos medir sus resultados e impactos?”. El profesional de la información, v. 16, n. 4, pp. 281-286.

https://doi.org/10.3145/epi.2007.jul.01

Charnes, Abraham; Cooper, William-Wager; Rhodes, Edwardo (1978). "Measuring the efficiency of decision making units". European journal of operational research, v. 2, n. 6, pp. 429-444.

https://doi.org/10.1016/0377-2217(78)90138-8

Chen, Tser-yieth (1997). "A measurement of the resource utilization efficiency of university libraries". International journal of production economics, v. 53, n. 1, pp. 71-80.

https://doi.org/10.1016/S0925-5273(97)00102-3

Chen, Yao; Morita, Hiroshi; Zhu, Joe (2005). "Context-dependent DEA with an application to Tokyo public libraries". International journal of information technology \& decision making, v. 4, n. 3, pp. 385-394. 
De-Carvalho, Frederico A.; José-Jorge, Marcelino; Filgueiras-Jorge, Marina; De-Oliveira-Medeiros, Renata (2013). "Análise de eficiência e desempenho no longo prazo: ilustração empírica de um modelo para avaliação de bibliotecas públicas". Investigación bibliotecológica: Archivonomía, bibliotecología e información, v. 27, n. 60, pp. 71-95. https://doi.org/10.1016/S0187-358X(13)72544-9

Elkins, Mark R.; Maher, Christopher G.; Herbert, Robert D.; Moseley, Anne M.; Sherrington, Catherine (2010). "Correlation between the Journal Impact Factor and three other journal citation indices". Scientometrics, v. 85, n. 1, pp. 81-93. https://doi.org/10.1007/s11192-010-0262-0

Faccin-Camargo, Bruna; Lobán-Acero, Lidia; García-Lacalle, Javier (2018). “Medición de la eficiencia en las bibliotecas de universidades públicas españolas". Revista gesto, v. 6, n. 1, p. 89.

https://doi.org/10.31512/rg.v6i1.2670

Fernández, Sergio; Rubio, Francisco (2013). “¿El dinero importa? Relación entre el presupuesto de la biblioteca y la productividad investigadora de la Universitat Politècnica de València". Revista española de documentación científica, v. 36, n. 4, e023.

https://doi.org/10.3989/redc.2013.4.1043

Hammond, Christopher J. (2002). "Efficiency in the provision of public services: A data envelopment analysis of UK public library systems". Applied economics, v. 34, n. 5, pp. 649-657.

https://doi.org/10.1080/00036840110053252

ISO (2014). ISO 11620:2014 - Information and documentation -- Library performance indicators.

https://www.iso.org/standard/56755.html

Lázaro-Rodríguez, Pedro; López-Gijón, Javier; Alonso, Sergio; Martínez-Sánchez, María-Ángeles; Herrera-Viedma, Enrique (2018). "Secaba-Rank, herramienta online para analizar y evaluar bibliotecas". El profesional de la información, v. 27, n. 2, pp. 278-288.

https://doi.org/10.3145/epi.2018.mar.06

Lázaro-Rodríguez, Pedro; López-Gijón, Javier; Herrera-Viedma, Enrique (2018). "Visibilidad de las bibliotecas públicas y la lectura en medios de comunicación españoles frente a otros hechos de la cultura y relación con su uso: medidas para su mayor promoción y difusión". BiD: textos universitaris de biblioteconomia i documentació, n. 40.

https://doi.org/10.1344/BiD2018.40.7

Lázaro-Rodríguez, Pedro; Vakkari, Pertti (2018). “Modelizando el uso de las bibliotecas públicas a través de sus características: estudio comparativo entre España y Finlandia para los préstamos y las visitas". Revista española de documentación científica, v. 41, n. 4, e216.

https://doi.org/10.3989/redc.2018.4.1544

Ling-Feng, Hsieh; Jiung-Bin, Chin; Mu-Chen, Wu (2014). "Cost efficiency and service effectiveness for university e-libraries in Taiwan". The electronic library, v. 32, n. 3, pp. 308-321.

https://doi.org/10.1108/EL-10-2011-0146

Liu, Shiang-Tai; Chuang, Mang (2009). "Fuzzy efficiency measures in fuzzy DEA/AR with application to university libraries". Expert systems with applications, v. 36, n. 2, Part 1, pp. 1105-1113.

https://doi.org/10.1016/j.eswa.2007.10.013

López-Gijón, Javier (2003). La red de lectura pública de Andalucía: un análisis sistémico. Tesis doctoral. Granada: Universidad de Granada.

http://digibug.ugr.es/bitstream/10481/29141/1/Lopez-GijonJavier.pdf

Luque-Martínez, Teodoro; Faraoni, Nina; Doña-Toledo, Luis (2018). “Meta-ranking de universidades. Posicionamiento de las universidades españolas". Revista española de documentación científica, v. 41, n. 1, e198.

https://doi.org/10.3989/redc.2018.1.1456

Martín-Martín, Alberto; Orduña-Malea, Enrique; Ayllón, Juan M.; Delgado-López-Cózar, Emilio (2016). “Un panorama académico de dos caras: retrato de los documentos altamente citados en Google Scholar (1950-2013)". Revista española de documentación científica, v. 39, n. 4, e149.

https://doi.org/10.3989/redc.2016.4.1405

Martin-Martin, Alberto; Orduña-Malea, Enrique; Harzing, Anne-Wil; Delgado-López-Cózar, Emilio (2017). “Can we use Google Scholar to identify highly-cited documents?". Journal of informetrics, v. 11, n. 1, pp. 152-163.

https://doi.org/10.1016/j.joi.2016.11.008

MECD (2018). Bibliotecas públicas españolas en cifras - Ministerio de Educación, Cultura y Deporte. http://www.mecd.gob.es/cultura-mecd/areas-cultura/bibliotecas/mc/ebp/portada.html 
Rebiun (2018). Estadísticas Rebiun. Red de Bibliotecas Universitarias Españolas.

http://rebiun.um.es

Reichmann, Gerhard (2007). “Measuring university library efficiency using data envelopment analysis”. Libri, v. 54, n. 2, pp. 136-146.

https://doi.org/10.1515/LIBR.2004.136

Reichmann, Gerhard; Sommersguter-Reichmann, Margit (2006). “University library benchmarking: An international comparison using DEA". International journal of production economics, v. 100, n. 1, pp. 131-147.

https://doi.org/10.1016/j.ijpe.2004.10.007

Simón-De-Blas, Clara; Arias-Coello, Alicia; Simón-Martín, José (2007). "Aplicación de la técnica DEA en la medición de la eficiencia de las bibliotecas de la Universidad Complutense de Madrid". Revista española de documentación científica, v. 30, n. 1, pp. 9-23.

https://doi.org/10.3989/redc.2007.v30.i1.366

Simon-Martín, José; Simon-De-Blas, Clara; Arias-Coello, Alicia (2011). “Changes in productivity of Spanish university libraries". Omega, v. 39, n. 5, pp. 578-588.

https://doi.org/10.1016/j.omega.2010.12.003

Simón-Martín, José; Simón-De-Blas, Clara; Arias-Coello, Alicia (2016). “Ranking de las bibliotecas universitarias españolas en la gestión del personal". Revista española de documentación científica, v. 39, n. 1, e119.

https://doi.org/10.3989/redc.2016.1.1259

Sin, Sei-Ching-Joanna; Vakkari, Pertti (2015). "Perceived outcomes of public libraries in the U.S." Library \& information science research, v. 37, n. 3, pp. 209-219.

https://doi.org/10.1016/j.lisr.2015.04.009

Stroobants, Jesse; Bouckaert, Geert (2014). "Benchmarking local public libraries using non-parametric frontier methods: A case study of Flanders". Library \& information science research, v. 36, n. 3, pp. 211-224.

https://doi.org/10.1016/j.lisr.2014.06.002

SYKT (2018). Suomen yleisten kirjastojen tilastot. Finnish Public Libraries Statistics Database.

https://tilastot.kirjastot.fi/?lang=en

Tavares, Rafael-Santos; Drumond, Geisa-Meirelles; Angulo-Meza, Lidia; Méxas, Mirian-Picinini (2018). "Efficiency assessment in university libraries". Transinformação, v. 30, n. 1, pp. 65-79.

https://doi.org/10.1590/2318-08892018000100006

Vakkari, Pertti; Aabø, Svanhild; Audunson, Ragnar; Huysmans, Frank; Oomes, Marjolein (2014). "Perceived outcomes of public libraries in Finland, Norway and the Netherlands". Journal of documentation, v. 70, n. 5, pp. $927-944$.

https://doi.org/10.1108/JD-06-2013-0072

Vakkari, Pertti; Aabø, Svanhild; Audunson, Ragnar; Huysmans, Frank; Kwon, Nahyun; Oomes, Marjolein; Sin, SeiChing-Joanna (2016). "Patterns of perceived public library outcomes in five countries". Journal of documentation, v. 72, n. 2, pp. 342-361.

https://doi.org/10.1108/JD-08-2015-0103

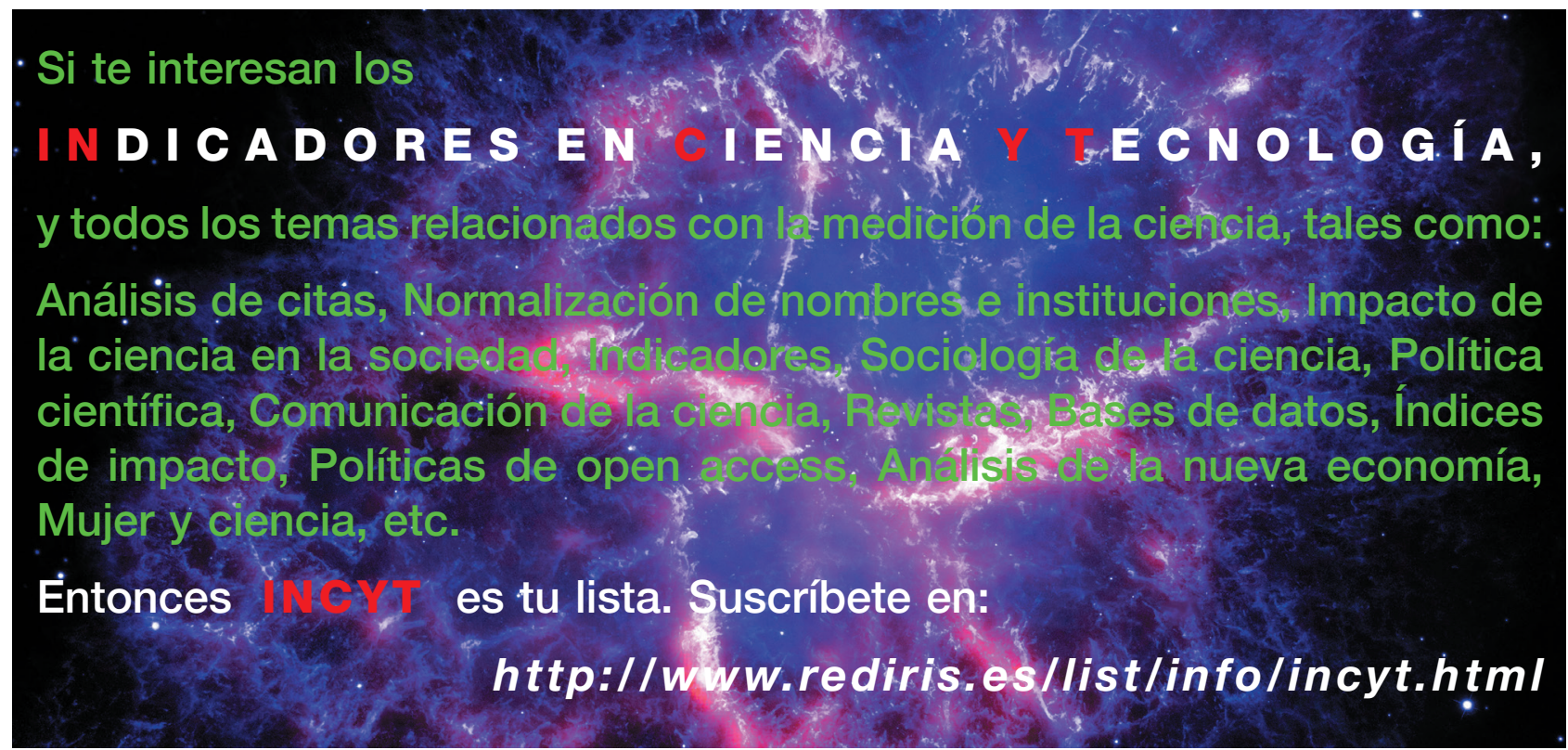

